\title{
Application of Remote Sensing Technology in Macro-Ecological Environment Monitoring
}

\author{
Miaocai Guo* \\ Hongyu Unmanned Aerial Vehicle Technology Co., Ltd. E-mail: miaocai@163.com
}

\begin{abstract}
At present, all countries in the world attach great importance to the development and application of remote sensing technology, which is widely used in many fields. By means of detection methods, this technology combines physics knowledge and modern network technology to provide relevant information for human exploration of geology, atmosphere, ocean and weather. In recent years, the global economy has developed rapidly. However, the environmental pollution has become increasingly serious at the same time. Industrial enterprises have discharged a large number of pollutants, resulting in air pollution, water pollution, soil pollution and so on, which seriously endanger human health and life safety. Environmental monitoring is the basis of effective control of environmental pollution. Remote sensing technology can be applied to carry out environmental monitoring and improve the monitoring effect of environmental monitoring.
\end{abstract}

Keywords: Remote Sensing Technology; Ecological Environment; Environmental Monitoring

\section{Introduction}

Remote sensing technology is a developing detection technology, which is widely used in many fields, especially in ecological environment monitoring. In recent years, the global environmental pollution has intensified, and the ecological environment in some areas has been seriously damaged. Remote sensing technology has gradually become an effective means to carry out ecological environment monitoring. Combined with the basic principle of remote sensing technology, this article discusses the application of remote sensing technology in macro-ecological environment monitoring, aiming to ensure better application of this technology in environmental monitoring.

\section{The basic principle of remote sensing technology}

Remote sensing technology is to detect and identify targets by sensing electromagnetic waves, visible light and infrared rays reflected by targets or radiated by themselves from a long distance. Remote sensing technology can be used to monitor air pollution, monitor the distribution of atmospheric molecules according to spectral principle, and find the source of air pollution. It can also be used to monitor water pollution and change of water environment, and to monitor soil pollution and understand the distribution of soil pollution in combination with vegetation cover and plant growth changes. The working principle of remote sensing instruments is complex. It can collect various kinds of information. When collecting information, remote sensing instruments will adjust the information collection mode according to the characteristics of the target areas. By flexibly using different remote sensing technologies,

\footnotetext{
Copyright (C) 2020 Miaocai Guo

doi: 10.18282/rs.v9i1.1099

This is an open-access article distributed under the terms of the Creative Commons Attribution Non-Commercial License

(http://creativecommons.org/licenses/by-nc/4.0/), which permits unrestricted non-commercial use, distribution, and reproduction in any medium, provided the original work is properly cited.
} 
more comprehensive information with a large amount of data can be collected, which is convenient for later analysis and comparison. Remote sensing instruments can work remotely, without field investigation, and can collect remote data to achieve the purpose of monitoring. At present, China's remote sensing technology is developing rapidly, with expanding information collection area and improving information collection efficiency. Remote sensing technology is an effective way to collect information, which can speed up information transmission, save time and improve efficiency. It can quickly grasp the ecological environment, provide data support for ecological environment governance, and promote ecological environment governance.

\section{Application of remote sensing technology in environmental monitoring}

\subsection{Air pollution}

Air pollution seriously affects human health. There are many sources of air pollution. Remote sensing technology can't measure the gas composition, but it can reflect the degree of air pollution by spectral principle. Specifically speaking, remote sensing technology can analyze air pollution according to the change range of near infrared band; it can carry out spectral analysis of atmospheric molecules to reflect the specific distribution of atmospheric molecules in the air; it can track the pollutants in the air in real time and make clear the distribution state of air pollutants, so as to carry out air pollution control.

\subsection{Water pollution}

Water is the basic resource for human survival, and also an important part of human body. Water pollution is extremely harmful to human health. Direct discharge of industrial wastewater and domestic sewage will pollute water sources and lead to water quality decline. At present, it is necessary to make rational application of remote sensing technology to monitor the water environment in real time. There are many rivers and lakes in China. Remote sensing technology can analyze water quality parameters and get the degree of water pollution by combining the changes of spectral signals.

\subsection{Soil pollution}

China's soil pollution is serious. Although remote sensing technology can't measure the content of heavy metals in soil, it can analyze soil moisture content, surface temperature, etc. to get the general condition of soil. Most plants are provided with nutrients by soil. Soil pollution seriously affects the quality of agricultural products and threatens human health. Therefore, remote sensing technology should be rationally applied to monitor the changes of soil environment. It can analyze the crops growing on each land, observe the growth and changes of crops, and at the same time use spectrum to grasp the distribution information of surface vegetation, rivers and buildings. Remote sensing technology is an effective method for soil environmental monitoring, which has wide coverage, fast information transmission and clear imaging, and can directly reflect the surface information. At present, China has established a satellite remote sensing monitoring platform to grasp the land pollution and improve the soil environment. This platform can investigate the specific situation of regional geographical environment, and pre-interpret and sketch the key survey features. For those cannot be interpreted on DOM, investigation and sketch on the spot are needed. This can greatly reduce the workload of the field survey, shorten the work cycle and improve the work efficiency. For example, if surveyors try to investigate the specific situation of soil pollution sources in the region, they can pre-read and mark the ground objects that may become pollution sources on DOM. After getting the general situation such as the specific location, they can design the survey route in a targeted way, and then go to the site for verification one by one, thus shortening the search time and improving the efficiency.

For some areas where the surface topography is complex or relatively dangerous, which makes it difficult for personnel to reach the field survey, unmanned aerial vehicles are used instead of personnel to carry out field survey, so as to obtain field information from DOM, thus reducing the risk of field survey work and improving the accuracy of data. The vector data of ground objects in the survey area can be obtained by vectorization of DOM, and the acquired data can be combined with GIS to process and analyze the vector data of ground objects, such as analyzing the distribution of forest land and 
cultivated land in the survey area, and mastering the real-time situation and important data of the ecosystem in the survey area from various aspects.

\subsection{The establishment of remote sensing monitoring system}

The remote sensing monitoring system is established based on multi-temporal DOM, which is used to monitor the change of surface features in the survey area. Based on the characteristics of DOM, such as strong real-time performance and large amount of information, the DOM in the investigation area is obtained at different time. Then, the monitoring data of ecological environment change in the investigation area are obtained by comparing and analyzing different temporal DOM, so as to control the change of ecological system in the investigation area macroscopically. For example, the DOM data of the survey area is acquired at the same time every year, and then the monitoring system is used for comparative analysis, so as to know the annual ecological changes of the area in this season. It provides scientific basis for environmental monitoring such as disaster's scale and loss assessment, land resource investigation, land reclamation and agricultural land remediation, forest data investigation, air pollution and water pollution. It also expands the breadth and depth of resource observation in ecological restoration investigation.

\subsection{The application of digital elevation model}

Digital Elevation Model (DEM) is a digital simulation of ground topography (that is, the digital expression of terrain surface morphology) through limited terrain elevation data. It is a solid ground model that uses a group of ordered numerical arrays to express ground elevation, and it is the digital expression of ground morphology, which contains abundant terrain and geomorphology information necessary for geoscience application analysis. DEM has many advantages, such as concise data structure, intuitive expression, simple and efficient interpretation of terrain factors. With the development of surveying and mapping technology in China, the research on DEM has been deepened gradually in recent years. Meanwhile, the digital terrain analysis technology based on DEM has become more mature, which plays an important role in the application of hydrogeology, surveying and mapping, geological disasters, land use and planning, etc. The application of DEM in ecological restoration investigation project of landscape forest field and lake grass project is as follows.

\subsubsection{Terrain analysis}

DEM is a spatial distribution model of actual topographic features, which intuitively and clearly reflects the topographic features in the survey area, including the spatial pattern of geomorphology and the spatial distribution of topography. The latest topographic data of the region can be obtained through DEM data based on the real-time characteristics. DEM is the basis of terrain analysis, from which the information of the surface features can be extracted for analysis, including contour line generation, terrain surface fitting, multi-scale topographic map and cross-section map making. In addition to the spatial analysis of geomorphic features, the terrain evolution in the survey area can be simulated by systematic analysis of multi-temporal DEM data. It can also analyze the inheritance characteristics of geomorphic evolution in the survey area and deepen the study of geomorphic evolution mechanism and development trend in the survey area.

Based on DEM, a watershed hydrological model for simulating river runoff in water area is constructed to extract the characteristic information of the watershed, including calculating the water flow direction, determining the drainage direction, calculating the watershed water conservancy characteristic value (area, length, etc.), dividing the sub-watershed and calculating the sub-watershed area, determining the river network structure, and calculating the watershed parameters. It is mainly used for terrain analysis and parameterization of the watershed, providing effective data support for hydrological simulation and analysis. With the support of GIS technology, digital elevation model can automatically extract the corresponding watershed hydrological information, which makes DEM widely used in the construction of watershed hydrological model, provides scientific basis for hydrological research in the investigation area, and improves the efficiency of watershed hydrological research.

\subsubsection{Land and climate research}

Slope, aspect and elevation are important 
topographic factors for studying land and climate, and play an important role in mountain ecological analysis and research. Terrain factors such as slope, aspect and elevation affect the differential distribution of solar radiation on the surface, which not only affects the spatial distribution of land use in local areas, but also affects the spatial distribution of meteorological and climatic elements in local areas. DEM is used as the core data for spatial analysis, and various topographic factors are extracted and analyzed, which provides scientific support for the research and analysis of soil erosion, distribution and land use status of sloping farmland, distribution characteristics of crops and biodiversity, and forest resources regulation and research. Many geological disasters (landslides, collapses, earthquakes, etc.) are closely related to topographic structures. Studying topographic factors in the survey area plays an important role in mastering the occurrence of geological disasters in the survey area. Taking landslides as an example, slope is one of the important internal factors for the development of many geological disasters. Most of the areas where landslides occur have higher slopes. Slope direction affects the regular differentiation of natural elements, thus affecting the development direction of landslides. Surface curvature affects the speed of soil erosion and deposition, and thus affects the speed of landslide development. To sum up, the extraction, analysis and monitoring of topographic factors based on high-precision DEM provide scientific basis and data support for the study of landslide development and distribution. At the same time, it can continuously monitor and analyze the development and occurrence of geological disasters in the investigation area based on multi-temporal DEM data, and further deepen the research on environmental changes and geological disasters.

\section{The significance of remote sensing technology in environmental monitoring}

At present, China's social economy is developing rapidly, with increasing population and consumption of resources, as well as tremendous pressure faced by the ecological environment. Remote sensing technology is an effective method to implement environmental monitoring, which can provide support for ecological environment management. By rationally applying remote sensing technology, people can better monitor ecological environment changes, deeply understand ecological environment pollution, evaluate ecological environment quality, relieve environmental pressure and finally protect ecological environment. Remote sensing technology can dynamically monitor the changes of ecological environment, so that the ecological environment control scheme can be scientifically formulated to improve the ecological environment control effect.

\section{Conclusion}

Air pollution, water pollution, soil pollution and other environmental problems seriously restrict the sustainable development of social economy. It is extremely important to reasonably apply remote sensing technology to macro ecological environment monitoring to provide information and data support for ecological environment pollution control. At present, remote sensing technology has been widely applied. It is necessary to constantly improve remote sensing technology and continuously develop and innovate relevant equipment, so as to give full play to the role of remote sensing technology, ensuring it to effectively improve the ecological environment.

\section{References}

1. Chen C. Evaluation of rural ecosystem services based on high-scoring remote sensing data-a case study of Deqing County, Zhejiang Province (in Chinese). Urban and Rural Construction 2020; (11): 26-29.

2. Lu T, Jin G, Zhang D. Study on evaluation of urban ecological environment quality in Ganzhou based on remote sensing ecological index (in Chinese). Electronic World 2020; (10): 96-97.

3. Yang Q, Zhang Q, Lu Y, et al. Ecological environment monitoring of Gannan Prefecture based on small unmanned aerial vehicle and remote sensing technology (in Chinese). Green Science and Technology 2020; (10): 143-147. 